\title{
Rural Health for Pregnant and Birthing People: Access and Advocacy
}

\author{
Sunday W. Smith and Erica Corbett ${ }^{1}$
}

Midwifery care is a safe and cost-effective care model; producing excellent outcomes especially for vulnerable and isolated people due to its one-on-one continuity of care and family-centered model. Recent research studies have shown out of hospital birth to have numerous benefits; shorter labors and lower rates of interventions, without an increase in adverse outcomes. These outcomes are more reliable when midwifery care is fully supported and integrated into existing maternity care systems. There are significant barriers to the provision of equitable reproductive health care to pregnant and birthing people in rural and remote areas. This, in the context of the United States being the only country where maternal mortality rates are on the rise. Midwifery care has been proposed as a potential solution but the need for working models of such care exists. The article showcases a working and replicable midwifery care practice model; showing how it can function to address inequity by building collaborative partnerships with other providers to advocate on clients' behalf and reduce vulnerability to health disparities. This has practice implications for maternity providers and policy makers, introducing how advocacy can help remove the systemic barriers affecting reproductive justice and care. [Article copies available for a fee from The Transformative Studies Institute. E-mail address: journal@transformativestudies.org Website: http://www.transformativestudies.org (C2021 by The Transformative Studies Institute. All rights reserved.]

KEYWORDS: Midwifery Care, Rural and Remote Health, Collaboration and Advocacy, Reproductive Justice, Out of Hospital Birth.

\footnotetext{
${ }^{1}$ Sunday W. Smith, MSN, APRN, CNM, is an adjunct Clinical Assistant Professor at Clarkson University. Moving from Indianapolis to Potsdam in 2017, Sunday W. Smith provides out of hospital Women's Health and Maternity care in this region's most rural and low-resource areas. She founded and is the director of a non-profit organization, North Country Baby Blessings, to raise funds to off-set the costs of this care. She is pursuing her Doctorate in Nursing Practice from University of Massachusetts to further prepare her to educate and advocate for access to woman-centered care. Address correspondence to: Sunday W. Smith, e-mail: swsmidwife@gmail.com.

Erica Corbett, MSc, CPM, Courtesy Affiliation with Department of Community Health and Epidemiology, Dalhousie University, Halifax, Nova Scotia, Canada. She is a midwife, reproductive health educator, and international consultant in maternal, neonatal and child health research. She is a passionate advocate for using evidence to improve access to high-quality care for birthing people and newborns and has over a decade of experience in sub-Saharan Africa. She obtained her Masters in Epidemiology and Community Health from Dalhousie University in 2010, and was awarded her midwifery certification through the North American Registry of Midwives (NARM) in 2018.
} 